Revista de Filología Alemana

ISSN: 1133-0406

https://dx.doi.org/10.5209/rfal.64355

\title{
¿Qué es un Funktionsverbgefüge? Propuesta de representación paradigmática a partir de la configuración de un ejemplar prototípico
}

\author{
María Egido Vicente ${ }^{1}$
}

Recibido: 4 de mayo de 2018 / Aceptado: 29 de junio de 2018

Resumen. A pesar de tratarse de un fenómeno lingüístico estudiando en profundidad por la germanística, la falta de consenso relativa a la delimitación paradigmática y sistematización de los Funktionsvergefüge (FVG) dentro del sistema general de la lengua alemana es una constante en la bibliografía especializada. Partiendo de esta premisa, el objetivo del presente trabajo consiste en la propuesta de un modelo de representación paradigmática de las construcciones a partir de la configuración de un ejemplar prototípico y su entorno periférico en consonancia con la naturaleza no discreta de los FVG como categoría lingüística integrante del aparato cognitivo general.

Palabras clave: Funktionsverbgefüge; parametrización; ejemplar prototípico; entorno periférico.

\section{[en] What is a Funktionsverbgefüge? Proposal of a Paradigmatic Representation Based on the Configuration of a Prototypical Example}

\begin{abstract}
Despite being a linguistic phenomenon studied in depth by the German linguistics, the lack of consensus regarding the paradigmatic delimitation and systematization of Funktionsvergefüge (FVG) within the general system of the German language is a constant in the specialized literature. Under this premise, the aim of this paper is to propose a model of paradigmatic representation of this type of construction based on the configuration of a prototypical example and its peripheral environment in line with the nondiscreet nature of FVG as a linguistic category that forms part of the general cognitive system.
\end{abstract}

Keywords: Funktionsverbgefüge; Parametrization; Prototypical example; Peripheral environment.

Sumario. 1. Introducción. 2. Reduccionismo versus dinamismo: implicaciones metodológicas en el tratamiento de los mecanismos morfosintácticos. 3. Exclusividad e interacción: dos formas de entender el paradigma de los FVG. 4. Hacia la configuración de un modelo de parametrización. 4.1 Punto de partida: das Modell der umrahmten Schnittmengen de Kamber (2008). 4.2 Atributos primarios y secundarios: caracterización del prototipo y su entorno periférico. 4.2.1. Atributos primarios. 4.2.2. Atributos secundarios. 5. Conclusiones.

Cómo citar: Egido Vicente, M., «¿Qué es un Funktionsverbgefüge? Propuesta de representación paradigmática a partir de la configuración de un ejemplar prototípico», Revista de Filología Alemana 27 (2019), 131-151.

\footnotetext{
$1 \quad$ Universidad de Salamanca (España)

E-mail: mariaegido@usal.es
} 


\section{Introducción}

La publicación del artículo de Gerhard Helbig (1979) "Probleme der Beschreibung von Funktionsverbgefüge im Deutschen" supone un punto de inflexión en el estudio de los FVG en lengua alemana. Tras el inciso generativista, materializado en las aportaciones de Herrlitz (1973) y Persson (1975), Helbig retoma la senda estructuralista iniciada en la década precedente ${ }^{2}$ con el fin de superar la función casi ornamental atribuida a este tipo de construcciones por parte de la estilística durante la primera mitad del siglo $\mathrm{XX}^{3}$.

Desde la perspectiva de la enseñanza del alemán como lengua extranjera (DaF), Helbig hace referencia a dos aspectos que dificultan la asimilación de construcciones del tipo zur Aufführung bringen por parte de los aprendices: por un lado, el hecho de que no siempre sea posible contar con un equivalente tipológico en otras lenguas y, por otro, la falta de una descripción gramatical sistematizada ${ }^{4}$. Bajo esta premisa, Helbig (1979: 274) describe una serie de propiedades básicas de los FVG, que, todavía hoy, son ampliamente aceptadas en el ámbito de la germanísticas: (i) entre el verbo y el sustantivo se establece una relación de interdependencia de la que resulta una unidad de significado, (ii) la construcción puede ser sustituida por un verbo pleno o un adjetivo + verbo copulativo de significado afín, (iii) el componente nominal aporta la carga léxica fundamental a la construcción, (iv) el componente verbal es un verbo funcional (en adelante VF) con una carga léxica debilitada respecto a su variante plena, (v) el sustantivo es un abstracto deverbal o deadjetival y expresa la carga léxica del verbo o adjetivo base de la derivación y (vi) la función principal de la preposición que introduce el componente nominal es la marcación de caso.

A pesar del alto grado de consenso respecto a los que pueden considerarse requisitos básicos de formación y de la existencia de una vasta bibliografía, la noción de FVG dista mucho de recibir una definición unánimemente aceptada. En lo que concierne al presente trabajo, evitamos participar en la manida discusión en torno a la definición canónica de los FVG y, así, en lo que Kamber (2008: 9) denomina "Definitionsakrobatik". Por otra parte, siendo conscientes de que cualquier formulación metodológica pasa por una delimitación previa del objeto de estudio, el objetivo de este trabajo consiste en el diseño de un modelo de representación paradigmática, que, tomando como punto de partida la percepción diferenciada e intuitiva de las

\footnotetext{
Véanse, entre otros, los trabajos de Engelen (1968) y Heringer (1968).

$C f$. Engel (1917), Weiskopf (1955) y Korn (1962). A principios de los años 60 se publican los primeros trabajos que legitiman la operatividad de los FVG en el sistema lingüístico alemán más allá de cuestiones estilísticas o idiolectales. Entre ellos, junto a Kolb (1962) y Daniels (1963), destaca la aportación de von Polenz (1963) en la que se llama la atención sobre la necesidad de llevar a cabo un análisis lingüístico alejado de los presupuestos teórico-metodológicos de la estilística para realizar una correcta valoración del fenómeno desde una perspectiva sincrónica. Anteriormente, Sütterling (1907: 179ss.) valora ya el fenómeno de los FVG en relación con la expresión de la Aktionsart, superando así su función como variante estilística de verbos plenos.

4 En la actualidad, el fenómeno lingüístico de los FVG cuenta con una amplia cobertura en las gramáticas contemporáneas de lengua alemana (cf. Zifonun et al. 1997: vol. 1 53-54 y 703-705 y vol. 2 1067-1068; Hentschel / Weydt 2013: 74-76; Eisenberg 2013: 305-312; Engel 2004: 210-211; Duden 2016: 425-433); sin embargo, en el momento de la publicación del artículo de Helbig todavía predomina el tratamiento estilístico, incluso en las gramáticas que recogían el fenómeno, generalmente de forma somera (véanse las primeras ediciones de la gramática Duden como ejemplo representativo: Duden (1959: 410ss./ 1966: 441ss./ 1973: 437ss.).

5 Helbig sintetiza aquí algunas de las características, que, de forma recurrente, sirven para la descripción del fenómeno en trabajos precedentes. Véase en esta relación los trabajos de Engelen (1968), Heringer (1968), Götze (1973), Starke (1975) y Günther / Pape (1976).
} 
construcciones en el plano cognitivo, resulte en la configuración del ejemplar prototípico de FVG a partir de la interacción de unos parámetros sistematizables.

\section{Reduccionismo versus dinamismo: implicaciones metodológicas en el tratamiento de los mecanismos morfosintácticos}

La falta de consenso en torno a la definición de los FVG se sucede en consonancia con las discrepancias relativas a su delimitación paradigmática y sistematización. Estas son, en parte, herederas del carácter multidisciplinar de este tipo de construcción verbonominal y, en mayor medida, de la aplicación de distintos enfoques y sistemas teóricos y metodológicos a su estudio a partir de una definición subjetiva del fenómeno, generalmente, desde un enfoque introspectivo ${ }^{6}$. En esta relación, tal y como afirma Detges (1996: 33), la valoración y estatus del paradigma de los FVG en el sistema lingüístico general de la lengua alemana están subordinados con frecuencia a los intereses de una corriente lingüística o metodológica determinadas ${ }^{7}$.

Si revisamos la bibliografía especializada, las divergencias conceptuales se articulan en torno a tres ejes fundamentales: la naturaleza desemantizada del VF, la relación parafrástica entre la construcción verbonominal y la variante plena y la delimitación del paradigma de los FVG frente al paradigma fraseológico. En el primer caso, Starke (1975: 159) apunta ya a la existencia de "auffallende Meinungsverschiedenheiten" entre aquellos que, como Bahr (1977: 29) y Götze (1979: 12), consideran el VF una forma gramaticalizada, próxima a los verbos auxiliares, y otros como Helbig (1979: 277-278) y von Polenz (1987: 171) que defienden su aportación sistemática de significado. La valoración del VF como un verbo vacío de significado implica la interpretación de la construcción verbonominal como variante estilística de la forma plena, limitando su función a la descomposición de una expresión de predicado simple en una expresión compleja (p.ej. zur Aufführung bringen $\rightarrow$ aufführen).

En cuanto a la relación con el paradigma fraseológico, se identifican igualmente posiciones encontradas. Entre otros, Rothkegel (1973: 50), Fix (1974-1976: 63) y Schemann (1982:84-86) identifican una extracción no compositiva del significado de la construcción verbonominal, situando los FVG en el sistema fraseológico. Por el contrario, en propuestas como las de Heringen (1968), Helbig (1979) y von Polenz (1987) los FVG se consideran objeto de estudio de la gramática.

Las discrepancias conceptuales en torno al fenómeno de los FVG se extienden también a los denominados “operationelle Kriterien” (Helbig 1979: 276278) relativos al comportamiento de las construcciones a partir de la realización de una serie de test de conmutación, permutación y paráfrasis en el marco de la metodología estructural-funcionalista. En base a estos y en contraposición a las combinaciones libres de palabras ofreie Wortverbindungen, la descripción de los FVG en lengua alemana aparece ligada tradicionalmente a la restricción de una serie de mecanismos morfosintácticos relacionados con la actualización, modifi-

\footnotetext{
Cf. Heine (2006: 12).

Un ejemplo representativo a este respecto es la aportación de Herrlitz (1973) centrado en ofrecer una visión crítica de la evolución de la teoría generativa hasta llegar a su variante semántica y no tanto en la descripción de la naturaleza de las construcciones. Los FVG se convierten aquí en el medio para llegar al fin, que no es otro que validar la semántica generativa como modelo de análisis lingüístico especialmente productivo en el estudio de los predicados nominales ( $c f$. Herrlitz 1973: X).
} 
cación y pronominalización del sustantivo, su operatividad como palabra referencial de una oración de relativo, la neutralización de la flexión de número y la pasivización ${ }^{8}$. En su formulación original, el artículo que introduce al sustantivo del componente nominal no puede ser intercambiado por otro artículo o determinante, de igual forma que también se neutraliza la alternancia sing./ pl. El sustantivo no admite ser sustituido por un pronombre, ni tampoco ser modificado por un adjetivo atributivo, un atributo en genitivo o un atributo preposicional. Por último, en esta relación, el núcleo del componente nominal no puede operar como palabra referencial de una oración de relativo, ni tampoco desempeñar la función de sujeto paciente. La aplicación de estos criterios, independientemente de la corriente lingüística subyacente, es un procedimiento frecuente en la descripción del fenómeno de los FVG en la germanística. Sin embargo, su aplicación admite diversas interpretaciones ${ }^{9}$ :

Tabla 1. Interpretación de las restricciones morfosintácticas en estudios específicos sobre $\mathrm{FVG}^{10}$.

\begin{tabular}{|c|c|c|c|c|c|c|c|}
\hline & Enfoque teórico & Act. & Núm. & Pro. & Mod. & Rel. & Pas. \\
\hline Daniels (1963) & $\begin{array}{c}\text { Inhaltsbezogene } \\
\text { Grammatik }\end{array}$ & - & frec & frec & - & frec & frec \\
\hline Heringer $_{1}(1968)$ & \multirow[t]{2}{*}{ funcionalismo } & + & + & & + & + & \\
\hline Heringer $_{2}(1968)$ & & - & - & & - & - & \\
\hline Herrlitz (1973) & semántica generativa & frec & & + & & + & \\
\hline Persson (1975) & semántica generativa & - & - & & - & - & \\
\hline Helbig $_{1}(1979)$ & \multirow[t]{2}{*}{ funcionalismo } & + & + & + & + & + & + \\
\hline Helbig $_{2}(1979)$ & & - & - & - & - & - & - \\
\hline von Polenz (1987) & semántica oracional & + & + & + & & & + \\
\hline Kamber (2008) & semántica de prototipos & - & - & - & - & & \\
\hline
\end{tabular}

Como ilustra la Tabla 1, la valoración irregular de estos mecanismos morfosintácticos no solo se pone de manifiesto entre trabajos realizados desde enfo-

8 Se trata de una selección representativa de los 16 criterios que Helbig (1979) propone en su trabajo con el fin de ilustrar las discrepancias en su aplicación.

9 Los trabajos seleccionados en la Tabla 1 configuran una muestra representativa de investigaciones que se ocupan del estudio de los FVG en el marco de la germanística. Para una exposición detallada de sus propuestas conceptuales y metodológicas desde un punto de vista contrastivo consúltese la monografía de Egido Vicente (2016).

10 Leyenda: Act. = actualización, Núm. = alternancia de número, Pro. $=$ pronominalización, Mod. $=$ modificación, Rel. = relativización, Pas. = pasivización. El símbolo “-” indica la desactivación de la restricción morfosintáctica y, por lo tanto, que se trata de un criterio no distintivo. El símbolo "+” indica que se mantiene la restricción y, por lo tanto, se trata de un criterio distintivo. Por otro lado, "frec" indica que, aunque se trata de una restricción frecuente, no constituye un criterio distintivo. El espacio en blanco indica que el autor no hace referencia explícita a esa restricción. 
ques teóricos diferentes ${ }^{11}$, sino que, como en el caso de Heringer (1968) y Helbig (1979), un mismo trabajo puede admitir varias interpretaciones. Esta condición se marca en la Tabla 1 con los subíndices $\mathrm{x}_{1} \mathrm{x}_{2}$ en las propuestas de Heringer (1968) y Helbig (1979). Con Heringer ${ }_{1}$ y Heringer ${ }_{2}$ nos referimos a la diferencia que el autor establece entre lo que denomina homogene Funktionsverbfügungen o construcciones del innerer Bereich y las construcciones pertenecientes al äußerer Bereich respectivamente (Heringer 1968: 53-54). De forma semejante, Helbig (1979: 279-280) diferencia entre eigentliche oder lexikalisierte bzw. idiomatische FVG (Helbig) y las denominadas uneigentliche oder nicht lexikalisierte bzw. nicht idiomatische FVG (Helbig ${ }_{2}$ ). Si tomamos como referencia la propuesta de este último, se observa como la interpretación de los criterios operacionales aquí contemplados se articulan en torno a dos polos opuestos: por un lado, una interpretación estricta o estrecha en la que los FVG se conciben como un fenómeno estático dentro del sistema lingüístico alemán y cuyo comportamiento morfosintáctico puede describirse a partir de una serie de parámetros estables independientemente del contexto en el que se realicen (Helbig ${ }_{1}$ ) y, por otro, una interpretación más laxa en la que las construcciones se presentan como un elemento dinámico que no siempre se comporta de la misma manera (Helbig ${ }_{2}$ ). En el primer supuesto estaríamos ante un caso de lo que Helbig (1979: 279) denomina "strukturalistischer Reduktionismus" en el que la metodología o enfoque teórico utilizado predomina sobre el propio objeto de estudio. En el segundo, por el contrario, el objeto de estudio predomina sobre la metodología como parte de un sistema en continua evolución (ibid.).

Estos dos enfoques metodológicos tienen una incidencia directa no solo en la concepción de los FVG, sino también en su delimitación paradigmática. Desde una perspectiva estrecha del fenómeno, los FVG conforman una clase cerrada dentro un paradigma autónomo en la que todos sus integrantes presentan un comportamiento homogéneo. Por otra parte, el estudio de las construcciones desde un enfoque amplio o no discreto admite la interacción del paradigma de los FVG con los paradigmas vecinos, tradicionalmente, las construcciones libres de palabras y las unidades fraseológicas, y, por lo tanto, la existencia de un comportamiento irregular de las construcciones a partir de un principio de gradación que no permite una delimitación axiomática del paradigma.

\section{Exclusividad e interacción: dos formas de entender el paradigma de los FVG}

El reduccionismo conceptual implica la expulsión del paradigma de aquellas construcciones que desactivan alguna de las restricciones morfosintácticas descritas arriba y, por lo tanto, demuestran un comportamiento irregular a partir de unos parámetros prefijados. De esta forma, los mecanismos morfosintácticos se convierten en lo que en analogía a Mendívil (1999: 254) puede denominarse "criterios de diagnóstico" que permiten la formulación de una serie de generali-

11 La diversidad conceptual que caracteriza la descripción del fenómeno de los FVG se pone de manifiesto con la imposibilidad de ilustrar en todos los casos la incidencia de los mecanismos morfosintácticos propuestos en términos absolutos. En su lugar se hace uso de formulaciones poco específicas, lo cual se representa en la Tabla 1 con la marca "frec." 
zaciones sobre el comportamiento del fenómeno lingüístico. Este planteamiento favorece los procesos de taxonomización y delimitación, lo que, por otra parte, sucede sin tener en cuenta el contexto lingüístico o situación comunicativa en los que se realizan las construcciones. Obsérvense a este respecto los siguientes ejemplos ${ }^{12}$ :

1. Die Wissenschaftler, die sich mit der Aggression beschäftigen, die von Haushunden ausgeht, sind längst zu einem anderen Ergebnis gekommen. (PLAN, 26/04/2010)

2. Und kam zu dem Ergebnis, dass es auf jedem Portal andere waren. (KULT, 26/05/2010)

3. Divergierten die grundsätzlichen Behandlungsziele (was bei den Patienten mit dem Therapieziel „,Lebensverlängerung” allerdings deutlich häufiger der Fall war!), neigten die Ärzte dazu, die konkrete Entscheidung zu treffen, ohne die Patienten einzubeziehen. (BIO, 26/09/2010)

4. Interessant ist dabei, ob bei einem im Hungerstreik ins Koma gefallenen Gefangenen sofort ein Betreuer beigeordnet werden muss, der dann ja auch die Entscheidungen zu treffen hätte. (BIO, 30/09/2010)

5. Jeder Nutzer entscheide individuell, ob eine Information wichtig oder relevant ist und leite sie daher per Retweet weiter. (NETZ, 06/05/2010)

6. Und zweitens wird die Google-Suche weniger wichtig, wenn die Nutzer direkt zur gewünschten Anwendung kommen, indem sie auf eine App tippen. (NETZ, 17/08/2010)

7. Das Bundesverwaltungsgericht hat vor fünf Jahren eine Entscheidung getroffen, die einen Weg ebnen könnte. (BIO1, 19/04/2010)

8. Es erscheint schon fraglich, ob bzw. inwieweit in solchen Konstellationen mit einer Bevollmächtigten überhaupt auf den mutmaßlichen Willen Bezug genommen werden muss (das Gesetz legt das allerdings nahe) - dafür gibt es ja eigentlich die Vollmacht. (BIO, 27/04/2010)

En los ejemplos (1) y (2) se muestra como la construcción zum Ergebnis kommen admite la alternancia entre el artículo determinado y el artículo indeterminado. De forma semejante, la construcción Entscheidung treffen permite la alternancia de flexión de número en los ejemplos (3) y (4), a la vez que admite ser modificada por un adjetivo atributivo en el ejemplo (3), algo que también sucede en los ejemplos (1) y (6), esta vez en las construcciones con componente nominal preposicional zu einem Ergebnis kommen y zur Anwendung kommen respectivamente. Retomando el caso de Entscheidung treffen, se observa también como en el ejemplo (7) el sustantivo de la construcción desempeña la función de palabra referencial de una oración de relativo, además de admitir la alternancia con el artículo indeterminado. Por último, en el ejemplo (8), el sustantivo de la construcción Bezug nehmen actúa como sujeto paciente. La

12 En lo sucesivo los ejemplos se extraen de un corpus elaborado en el marco de mi tesis doctoral sobre la modificación del evento de los FVG compuesto por blogs de carácter divulgativo sobre ciencia y tecnología integrados en diferentes periódicos digitales. La información entre paréntesis se corresponde con las abreviaturas de los blogs (PLAN= Planckton: http://blogs.faz.net/planckton/, KULT= Kulturkampf: https://blog.zeit.de/kulturkampf/, $\mathrm{BIO}=$ Biopolitik: http://blogs.faz.net/biopolitik/ y NETZ= Netzökonom: https://netzoekonom.de/) y la fecha de publicación de la entrada de la que se extrae cada muestra. 
neutralización de las restricciones morfosintácticas hace que, desde una perspectiva estrecha del fenómeno lingüístico, no se pueda sistematizar estas construcciones en el paradigma de los FVG, pero ¿qué son entonces?, ¿a qué paradigma pertenecen?, ¿dónde se ubican dentro del sistema general de la lengua alemana?

La posibilidad que se contempla bajo este enfoque metodológico consiste en integrar las construcciones dentro del paradigma de las combinaciones libres de palabras, lo cual no está exento de controversia. Con el fin de ilustrar este argumento tomamos como referencia la construcción del ejemplo (6) zur Anwendung kommen. Cualquier usuario competente de la lengua alemana con un mínimo de intuición lingüística identificaría alguna diferencia, al menos a nivel cognitivo, entre esta construcción y otras como zum Unterricht kommen, en la que el verbo funciona también acompañado de un sustantivo introducido por una preposición. La percepción diferenciada de las construcciones, tipológicamente afines en su estructura superficial $\left(\mathrm{V}+\mathrm{SP}_{[\text {Dat. }]}\right)$, responde a una motivación más semántica que morfosintáctica.

El verbo kommen presenta una carga léxica diferenciada en combinación con el sintagma zur Anwendung, que, además, incide en el proceso de selección argumental. Mientas que en una construcción como zum Unterricht kommen la realización semántica del sintagma preposicional contiene la marca [+ Ort $\left._{(\mathrm{dir})}\right]$, en zur Anwendung kommen esta marca desaparece. El sintagma zur Anwendung no es un lugar ni un destino, sino una acción, das Anwenden, lo que se podría representar con la marca [+ Handlung $\left.{ }_{(\text {anwenden }}\right]$, realizado morfológicamente por un sustantivo deverbal en función de núcleo del sintagma preposicional. Teniendo en cuenta estos dos ejemplos se observa que en el caso de zur Anwendung kommen el verbo pierde su capacidad para seleccionar complementos circunstanciales de dirección y, por lo tanto, parte de su carga léxica original como verbo de movimiento:

kommen <st. V.; ist> [mhd. komen, ahd. koman, gemeingerm. Wort]: 1. sich auf ein Ziel hin bewegen [u. dorthin gelangen]; anlangen, eintreffen [...] 20. verblasst in festen Verbindungen mit Verbalsubstantiven zur Umschreibung des entsprechenden Verbs (bes. des Passivs): zu Fall k.; zum Einsatz k.; zur Anwendung, zur Aufführung k. (DUW)

Como se extrae de la primera acepción del DUW, el valor semántico del verbo kommen se relaciona con la expresión de un movimiento; mientras que en la última (acepción 20) se hace referencia explícita al debilitamiento de su carga léxica en combinación con un sustantivo deverbal y, con ello, a lo que Helbig (1979: 279) denomina Grammatikalisierungsprozeß mental del verbo kommen se limita aquí a la expresión de la Aktionsart $^{13}$. Este carácter debilitado del verbo implica un mayor protagonismo de la carga semántica aportada por el sustantivo deverbal en el proceso de decodificación, que, por otra parte, necesita del verbo debilitado o funcional para realizarse. De esta forma, el sustantivo expresa la acción o el evento (das Anwenden) mientras que el verbo especifica el punto de su realización (Aktionsart incoativa). Esta relación de

13 En relación a la expresión de la Aktionart como función semántica fundamental del VF véanse, entre otros, Heringer (1968: 81), Engelen (1968: 81), Helbig (1979: 281) y von Polenz (1987: 172ss.). 
dependencia mutua que se establece entre el verbo debilitado semánticamente y el sustantivo hace que la construcción se perciba con un grado mayor de fijación que las denominadas combinaciones libres de palabras, como por ejemplo zum Unterricht kommen y, así, en consonancia con el grado de gramaticalización del verbo funcional, como una construcción en proceso de lexicalización ${ }^{14}$. La pregunta que surge en esta relación es si ese grado de fijación justifica una sistematización de la construcción en el paradigma de las unidades fraseológicas. Para ilustrar esta posibilidad contraponemos la construcción zur Anwendung kommen a otras del tipo zur Welt kommen.

Desde el punto de vista de la percepción, el usuario de la lengua alemana se encuentra de nuevo ante dos construcciones que comparten estructura superficial, en la que el verbo kommen combina con un sintagma introducido por la preposición $z u$. Otro aspecto que tienen en común en contraposición a una construcción como zum Unterricht kommen es el grado de fijación. La diferencia radica en el proceso de decodificación, más complejo en el caso de zur Welt kommen por la naturaleza no compositiva de su significado o, lo que en analogía a Lipka (1992: 7) puede entenderse como grado de lexicalización. De esta forma, aunque el usuario conozca el significado de todas las palabras que componen la construcción, tendrá que llevar a cabo un proceso de abstracción hasta llegar al significado translaticio de zur Welt kommen, "geboren werden", un proceso que no tiene que realizar para la decodificación de la construcción zur Anwendung kommen $^{15}$.

Si tenemos en cuenta los supuestos anteriores se observa que el comportamiento morfosintáctico de las construcciones no parece ser suficiente para justificar su pertenencia al paradigma, sino más bien, tal y como afirma Kamber (2008: 29), "die Festigkeit der jeweiligen Syntagmen" ${ }^{16}$. Partiendo de esta premisa y con el fin último de sistematizar los FVG dentro del paradigma, algunos estudiosos del fenómeno, entre otros Daniels (1963: 27), Helbig (1979: 278-280) y Heringer (1968: 74), desde una perspectiva amplia, han tratado de formalizar el grado de operatividad de estos criterios morfosintácticos en cada una de las construcciones. Para ello, se recurre con frecuencia a la división del paradigma en un centro y una periferia. Tradicionalmente, las construcciones que presentan un comportamiento regular y, por lo tanto, activan las restricciones de carácter morfosintáctico, son consideradas construcciones prototípicas y se sitúan en el centro del paradigma. Por el contrario, las construcciones que admiten alguna alteración son consideradas construcciones periféricas ${ }^{17}$.

14 Con esta afirmación se hace referencia a la correspondencia que establece Helbig (1979: 279) entre el grado de gramaticalización del VF y el grado de lexicalización del FVG, de la cual, como señala el autor, depende además el grado de estabilidad semántica y morfosintáctica de la construcción: "Dem Prozeß der Grammatikalisierung der FVG entspricht der Prozeß der Lexikalisierung der gesamten FVG: Indem die FV einem höheren Grammatikalisierungsgrad erreichen, vergrößert sich die Festigkeit und die semantische Stabilität der FVG. Je weiter dieser Prozeß fortgeschritten ist, umso mehr erfüllen die FVG die gennanten operationellen Kriterien".

15 Aparentemente, este proceso mental no es tan obvio en un hablante nativo de lengua alemana. Sin embargo, si el usuario de la lengua es un aprendiz de alemán como lengua extranjera esta dificultad en el proceso de decodificación se acentúa.

16 Kamber (2008: 29) diferencia aquí entre lo que denomina Definitionskriterien y Entdeckungsprozeduren, refiriéndose con estos últimos a las restricciones morfosintácticos arriba descritas ( $c f$. Tabla 1).

17 Este enfoque metodológico que diferencia entre FVG prototípicos y periféricos cuenta con un amplio grado de aceptación en la germanística. Véase, por ejemplo, la última edición de la gramática Duden (2016: 425-426). 
Bajo esta premisa, y a diferencia de lo que sucede desde el enfoque reduccionista, los mecanismos morfosintácticos se valoran de forma gradual. Se admite la existencia de un continuum, y, por lo tanto, tal y como se ilustra en la Imagen 1, de zonas de transición entre el centro y la periferia, así como entre el paradigma de los FVG y los paradigmas vecinos:

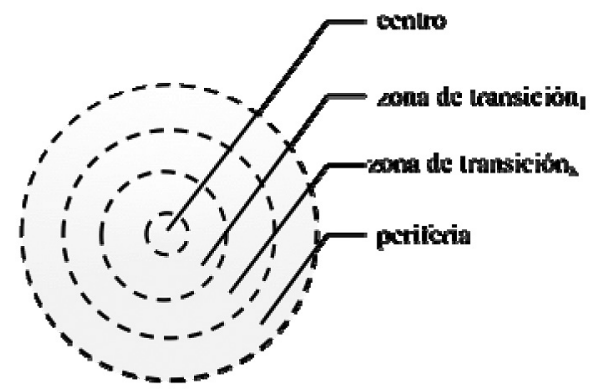

Imagen 1. Centro y periferia del paradigma de los FVG desde una perspectiva amplia.

Este enfoque permite sistematizar un amplio número de construcciones; sin embargo, al igual que sucede con el enfoque reduccionista, parte de una serie de criterios formulados a priori para distribuir las construcciones dentro del paradigma; es decir, previos a la observación del comportamiento de las construcciones en un contexto comunicativo determinado. Desde nuestro punto de vista y en consonancia con Kamber (2008: 22), aunque reconocemos su valor para poner de manifiesto diferencias o variaciones en la realización morfosintáctica de las construcciones, dudamos del grado de adecuación de este tipo de mecanismos como parámetros de delimitación.

Estos criterios describen el comportamiento de las construcciones en un contexto lingüístico o estadio de la lengua determinados ${ }^{18}$, pero aportan escasa información sobre características intrínsecas a las construcciones independientemente de su grado de proximidad o lejanía a las combinaciones libres de palabras o unidades fraseológicas.

\section{Hacia la configuración de un nuevo modelo de parametrización}

A continuación, proponemos un modelo de parametrización de los FVG que trata de superar la aplicación más o menos restrictiva de una serie de mecanismos morfosintácticos como criterios definitorios o delimitadores, tal y como se plantea en los estudios tradicionales. Para ello, en analogía a la propuesta de Kamber (2008), partimos de los principios de la semántica de prototipos con el fin de poder ofrecer una delimitación del fenómeno lingüístico que respete su carácter heterogéneo

18 En este punto nos referimos a que el fenómeno de los FVG pueda estar sujeto a variaciones sincrónicas y diacrónicas, lo cual, en ocasiones, explicaría su comportamiento morfosintáctico más o menos regular. En esta relación, véanse las aportaciones de Relleke (1974) y Blum (1986). 
y multidisciplinar, así como la propia idiosincrasia del sistema lingüístico en el que se realiza, en este caso la lengua alemana.

\subsection{Punto de partida: das Modell der umrahmten Schnittmengen de Kamber (2008)}

Nuestra propuesta se articula en torno a tres premisas conceptuales en consonancia con una concepción amplia del fenómeno lingüístico ( $c f$. Imagen 1) y la aplicación del marco teórico de la semántica de prototipos al estudio de los FVG (Kamber, 2008). En primer lugar, que los FVG configuran una clase heterogénea y que, por lo tanto, no es posible llevar a cabo una distribución homogénea de las construcciones dentro del paradigma. En segundo lugar, que se trata de un paradigma productivo en el que se diferencia entre un centro y una periferia y que, además, permite la interacción con los paradigmas vecinos ${ }^{19}$, así como la incorporación de nuevas estructuras $\mathrm{y}$, en tercer lugar, el hecho de que, como afirma Lakoff (1987: 67), los FVG, como estructuras lingüísticas y parte integrante del aparato cognitivo general, son susceptibles de someterse a una categorización prototípica.

En el apartado 3 hemos hecho referencia a la percepción diferenciada que un usuario competente de la legua alemana puede experimentar de construcciones con una estructura superficial semejante del tipo zur Anwendung kommen, zum Unterricht kommen y zur Welt kommen. En caso de tratarse de un hablante nativo, este proceso cognitivo se desarrolla de forma intuitiva. En nuestra propuesta de parametrización, partiendo de este supuesto, se pretende precisamente, tal y como expone Moure (1994: 202) "traducir ese valor intuitivo a parámetros sistematizables" con el fin de configurar el ejemplar prototípico de FVG. Para ello, tomamos como punto de partida el trabajo de Kamber (2008) y su modelo de inserción de conjuntos o Modell der umrahmten Schnittmengen, para cuya configuración adapta los presupuestos de la semántica de prototipos al estudio de 1 os $\mathrm{FVG}^{20}$. Su objetivo es poner a disposición del usuario, en este caso aprendices de alemán como lengua extranjera, un inventario de construcciones representativo ${ }^{21}$ a partir de un ejemplar prototípico, que, en base a la propuesta de Kleiber (1990), define de la siguiente manera ${ }^{22}$ :

Als Prototyp bezeichnet man in der Prototypentheorie die Kombination von typischen Merkmalen einer Kategorie; der Prototyp erscheint dort, wo die Überlappung am ausgeprägten ist. Es kann sich auch um eine Art “idealen Typ” der Kate-

19 La interacción entre los paradigmas ha de entenderse aquí desde una persectiva diacrónica y en relación a los procesos de lexicalización. En esta relación, consúltese Daniels (1963:26) y Persson (1975:15).

20 Kamber parte aquí de un trabajo precedente (Kamber / Näf, 2001) en el que se introduce la noción de ejemplar prototípico al estudio contrastivo de los FVG para el par de lenguas alemán- francés. Posteriormente, aunque de forma sucinta, Seifert (2004: 52-53) hace también referencia a la semántica de prototipos para delimitar su objeto de estudio: los FVG en textos jurídicos alemanes entre los siglos XVIII y XX.

21 En el trabajo de Kamber la representabilidad es sinónimo de frecuencia (2008: 4).

22 Para el desarrollo de su modelo Kamber parte de la versión ampliada de la teoría de los prototipos formulada por Kleiber (1990: 147-187) a partir del concepto de Familienähnlichkeit de Wittgenstein en la que se abandona la idea central de prototipo tal y como propone Rosch (1973) en la que se conoce como versión estándar de la teoría de los prototipos. Con el fin de obtener una visión panorámica del concepto de prototipo en sus diferentes versiones consúltese, entre otros, el trabajo de Moure (1994). 
gorie (Kleiber 1990: 185ff.) im Vergleich zu anderen Exemplaren handeln, die je nach ihrer Ähnlichkeit mit dem Prototyp mehr oder weniger weit entfernt von Zentrum angeordnet sind. (Kamber 2008: 22-23)

Partiendo de esta concepción de prototipo, Kamber (2008: 21) propone los siguientes cuatro criterios con el fin de obtener el mejor representante de la categoría:
[A] El verbo del FVG es un VF
[B] ¿Es el sustantivo un sustantivo abstracto deverbal?
[C] ¿Es el VF un verbo de movimiento o estado?
$[\mathrm{D}]$ ¿Contiene el sintagma un grupo preposicional?

El criterio [A] o Basis-Kriterium es el único que presenta una naturaleza restrictiva y que, en consecuencia, se manifiesta en todas las construcciones. La concepción de VF de Kamber se aproxima aquí a la de Helbig (1979: 274) y, por lo tanto, a la de un verbo con una carga léxica debilitada que necesita de un sustantivo para expresar un predicado, pero que, en ningún caso, se presenta como una forma gramaticalizada en su totalidad. A pesar de esta aproximación conceptual, Kamber no delimita la noción de VF al entender que el grado de desemantización de un verbo no admite una interpretación binaria, lo que, tal y como afirma el autor, constituye un punto débil en su modelo de parametrización:

Die Achillesferse dieses Modell ist eindeutig, dass wir es mit einem „weichen“ Grundkriterium zu tun haben: Manche Verben haben neben einer „volleren“ auch eine „verblasstere“ Bedeutung. Doch: Ist die Zuteilung zu den FV bloß Ermessenssache oder gibt es eine „Messlatte“? (Kamber, 2008: 23).

En relación al criterio [B], Kamber cuestiona las nociones de nomen actionis y Verbalabstraktum, que, tradicionalmente ${ }^{23}$, sirven para caracterizar el sustantivo de la construcción verbonominal. En consecuencia, en analogía a Bresson (1989), destaca la función predicativa del sustantivo de las construcciones en detrimento de su naturaleza morfológica como forma derivada de un verbo o adjetivo. Para la formulación del criterio [C] Kamber parte de la categorización semántica de los verbos funcionales como verbos de estado o movimiento a partir de la propuesta de Gerling / Orthen (1979: 99ss.), diferenciando, en este último caso, entre un aktive Eigenbewegung/aktive Fortbewegung en verbos del tipo kommen y un passive Fortbewegung en verbos del tipo führen. En cuanto al criterio [D], Kamber propone una delimitación dicotómica a nivel morfológico que afecta a la realización del componente no verbal de la construcción en forma de grupo nominal o grupo preposicional.

23 Cf. Heringer (1968), Helbig (1979), Götze (1979), von Polenz (1987), Rösch (1994) y Seifert (2004). 
La combinación de estos criterios deriva en la configuración del siguiente modelo de parametrización:



Imagen 2. Distribución de los FVG en el modelo de inserción de conjuntos de Kamber (2008:22).

(1) zum Ausdruck kommen; in Aufregung geraten

(2) Stellung nehmen

(3) zur Verfügung haben; in Besitz haben

(4) zur Welt kommen; zu Papier bringen

(5) Zurückhaltung üben; Wache halten; Anwendung finden

(6) in Angst halten

(7) Platz nehmen

(8) Lust haben/ ein Foto machen

(9) zur Geburtstagsfeier gehen/Zucker nehmen/Kuchen essen

Como se observa en la Imagen 2, los FVG se distribuyen en torno a un ejemplar prototípico. Se trata de las construcciones integradas en el grupo (1) en las que, además del criterio básico de formación [A], se presentan los atributos prototípicos formulados en el resto de criterios. De esta forma, en la propuesta de Kamber, el mejor representante de la categoría de los FVG es una construcción en la que un VF, en su origen un verbo de estado o movimiento, combina con un grupo preposicional cuyo núcleo es un sustantivo en función predicativa.

\subsection{Atributos primarios y secundarios: caracterización del prototipo y su entorno periférico}

En nuestra propuesta, al igual que en la de Kamber (2008), diferenciamos entre lo que hemos denominado atributos base o primarios, operativos en todas las construcciones como requisitos básicos de formación, y unos atributos secundarios que pueden o no manifestarse. A continuación, proponemos una descripción detallada de cada uno de ellos:

\subsubsection{Atributos primarios}

[A] El componente verbal es un VF: un VF es, por definición, un verbo que experimenta una pérdida de su carga léxica original. Como indica Kamber (2008: 23), 
este proceso solo admite una interpretación gradual, lo cual, por otra parte, no implica que la categoría de VF no pueda ser objeto de análisis. En este planteamiento nos basamos en Wierzbicka (1989) y en su crítica al uso generalista de la noción de prototipo en el marco de la teoría semántica. Como afirma en su estudio, "concepts encoded in natural language, are, in a sense, vague, but this does not mean that their semantic description should be vaque too" (1989: 365). Esta premisa conceptual motiva la formulación de la siguiente pregunta en relación a nuestro objeto de estudio: ¿es posible describir el VF más allá de su grado de desemantización? Con este propósito, partimos, en primer lugar, de la contextualización de la categoría de los VF en el sistema verbal alemán entre la categoría de los verbos plenos y los verbos auxiliares (Helbig, 1979: 274). Por otra parte, como consecuencia de la debilitación de su significado, el VF pierde la capacidad de completar su estructura argumental y, por lo tanto, de expresar un predicado. Para ello necesita la carga léxica de un sustantivo con el que no mantiene una relación argumental según las reglas generales de la gramática, ya que, en este caso, no es el verbo el que selecciona al sustantivo, sino el sustantivo el que selecciona al verbo con el fin de enmarcar la expresión de predicado en unas coordenadas espacio-temporales ${ }^{24}$.

Junto a esta caracterización general proponemos los siguientes principios, que, aunque también derivados del proceso de desemantización, se aplican de forma independiente a su grado de desarrollo: principio de implicación restringida, principio de no conmutación y principio de no coocurrencia. El primero alude a la relación establecida entre la variante funcional y la variante plena de un verbo, de manera que la operatividad de la primera en el sistema lingüístico implica la existencia de la segunda, no al revés ${ }^{25}$. Por otra parte, según el principio de no conmutación, el VF no puede conmutar con variantes plenas independientemente del contexto en el que se realice. De esta forma, el VF operativo en la construcción zur Anwendung kommen no puede ser sustituido por verbos afines semánticamente a su variante plena, como, por ejemplo, sich einfinden (*sich zur Anwendung einfinden $)^{26}$. Por último, en consonancia con el principio de no coocurrencia, la variante funcional y la variante plena no pueden activarse simultáneamente en una misma unidad funcional. Se trata de un parámetro especialmente pertinente en el caso de los FVG con componente nominal en acusativo en relación a ejemplos como el siguiente:

(9) Gerade die Veranstalter wissenschafticher Kongresse haben - so weit ich es bislang erlebt habe - auch kein besonderes Interesse und vor allem auch kein Geld die Kosten der Medienvertreter zu übernehmen. (BIO24, 26/09/2010)

La variante funcional y plena del verbo haben parecen ocurrir de forma simultánea con el sustantivo Interesse y Geld respectivamente. Desde nuestro punto de vis-

24 Cf. Alonso Ramos (2004: 87).

25 Aunque se trata de un supuesto ampliamente aceptado en el ámbito de la germanística, en algunas investigaciones sobre FVG en otras tradiciones lingüísticas se plantea la posibilidad de la existencia de VF sin variante plena, así como de VF semánticamente plenos. Se trata, entre otros, de los trabajos de Reuther (1996) para el ruso y Alonso Ramos (2004) para el español, ambos bajo un enfoque lexicográfico.

26 Cf. Helbig (1979: 276). Compárense con la forma plena del verbo en construcciones como zum Unterricht kommen en las que sí es posible la conmutación (sich zum Unterricht einfinden). 
ta, sin embargo, este ejemplo no ha de interpretarse como un caso de bifuncionalidad de la unidad léxica, sino como una elisión motivada por la equivalencia de forma entre la variante plena y la variante funcional del verbo ${ }^{27}$.

[B] El componente nominal está formado por un sustantivo que expresa un evento: con el fin de delimitar el sustantivo tipo que opera en un FVG partimos de una perspectiva pluridimensional en el que se tienen en cuenta parámetros de orden morfológico, sintáctico y semántico. Como se indica en la descripción del atributo primario [A], el VF necesita un sustantivo para realizar la expresión de predicado, lo cual pone de manifiesto su valor predicativo en detrimento de su valor referencial ${ }^{28}$. Partiendo de esta premisa, el sustantivo, en su función predicativa, participa en la expresión del evento descrito por el predicado que conforma junto al VF, el cual, en analogía a de Miguel (1999), entendemos de forma amplia:

Con el término evento se alude a cualquier tipo de 'situación' o ‘acontecimiento' denotado por un predicado. [...] Evento engloba, pues, acciones (acontecimientos llevados a cabo voluntariamente por un sujeto agente), procesos (acontecimientos desencadenados espontáneamente o causados por una fuerza mayor externa al proceso) y estados (situaciones que se mantienen a lo largo de un periodo) (de Miguel 1999: 1281).

De esta definición se extrae una caracterización ternaria de la noción de evento en acciones, procesos y estados que permite superar la problemática en torno a la identificación del sustantivo de la construcción con un nomen actionis. En esta relación y en consonancia con Heringer (1968: 26-28) consideramos que la ambigüedad semántica entre los nomen actionis y nomen acti no es pertinente para la descripción del ejemplar prototípico de $\mathrm{FVG}^{29}$. Se trata de sustantivos de carácter polisémico del tipo Einfluss que admiten una doble interpretación como acción o resultado/producto de una acción realizada previamente. Más compleja parece la interpretación de sustantivos en los que la contraposición acción-resultado/producto presenta un mayor grado de transparencia, como por ejemplo Anzeige. En este caso la oposición acciónresultado/producto se sucede en consonancia con la oposición inmaterial-material, como por ejemplo en la construcción eine Anzeige stellen (acción/ inmaterial) frente a eine Anzeige lesen (producto/ material). Algo semejante sucede también con los denominados nomina qualitatis y nomina essendi que denotan cualidades, sentimientos o estados. Obsérvese a este respecto el siguiente ejemplo:

(10) Andere Hunde, etwa Setter, Pudel, Collies, haben auf dem Behandlungstisch Angst. (PLAN6, 26/05/2010)

El sustantivo Angst denota el evento que pone de manifiesto el sentimiento expresado por ese mismo sustantivo en una oración como Angst ist ein Grundge-

\footnotetext{
Cf. Castell (1999: 130).

Cf. Koch (1981: 76-77) y Detges (1985: 80-82).
}

29 En analogía a la propuesta de delimitación de Porzig (1930: 72) para los sustantivos concretos y abstractos, Heringer (1968: 26) considera que no es posible delimitar formalmente los nomina actionis de los nomina acti, sino tan solo diferenciarlos a nivel contextual. En su propuesta, nomina acti como Sprache o Rede son interpretados como nomina actionis en combinación con un verbo funcional (zur Sprache kommen/ zur Rede stellen). 
fühl. En el ejemplo (10), al igual que Anzeige en construcciones como eine Anzeige stellen, el sustantivo Angst participa en la caracterización semántica de la estructura argumental del predicado; es decir, aporta la valencia semántica, lo cual implica un debilitamiento de su carácter referencial en contraposición a nombres puramente designativos. Estos sustantivos son susceptibles de formar parte del componente nominal de un FVG, ya que admiten una interpretación eventiva como resultado de un proceso de reinterpretación de un sentimiento (Angst) o un producto (Anzeige) en un determinado contexto sintáctico, como, en el caso que aquí nos ocupa, en combinación con un VF. Por esta razón, en vez de relación metonímica o de cambio semántico en sentido estricto, hacemos aquí referencia a un caso de polisemia ${ }^{30}$.

\subsubsection{Atributos secundarios}

[C] El componente verbal tiene un significado general o taxonómico: este atributo alude a la caracterización semántica del ejemplar prototípico de VF. Este tipo de verbos, tal y como describe el atributo primario [A], presentan una carga léxica debilitada como resultado de un proceso de desemantización, que, en su etapa más avanzada, solo permite la expresión de significados generales o taxonómicos en torno a cuatro categorías semánticas: causatividad, incoatividad, duración y pasividad ${ }^{31}$ (von Polenz, 1987: 172-174). Se trata de la aportación semántica mínima y constante de los VF a la construcción. Prueba de ello es la configuración de las denominadas series por conmutación o Reihenbildungen ${ }^{32}$ del tipo in Bewegung setzen (causativo)- in Bewegung kommen (incoativo)- in Bewegung sein (durativo), en las que el proceso de desambiguación léxica de FVG con un mismo componente nominal se realiza a partir de la aportación semántica del VF. Los verbos que presentan un significado taxonómico o general favorecen la formación de este tipo de series y presentan una mayor productividad combinatoria.

En ocasiones, estos verbos pueden ser sustituidos por otros, que, aunque también funcionales, presentan un mayor grado de especialización semántica, como por ejemplo ausüben y liefern en construcciones como Druck ausüben e Information liefern en contraposición a machen y geben respectivamente (Druck machen e Information geben). En nuestro modelo de parametrización, los FVG en los que operan este tipo de verbos se sitúan en el entorno periférico del paradigma.

[D] El componente nominal está formado por un sustantivo que mantiene una relación semántica con un verbo o adjetivo de base léxica común: tradicionalmente, la función predicativa del sustantivo operativo en este tipo de construcciones se pone en relación con su carácter abstracto en contraposición a lo que comúnmente se entiende por sustantivo concreto ${ }^{33}$. En nuestra propuesta preferimos prescindir de la oposición abstracto-concreto para delimitar la noción de sustantivo

Cf. Duden-Grammatik (2016: 739-740).

31 Von Polenz se refiere aquí a FVG como Anerkennung finden que realizan un predicado pasivo y no a los passivwertige Fuktionsverbgefügen de Rösch (1994) del tipo x bringt y zur Entscheidung en los que FVG causativos, incoativos o durativos denotan un significado pasivo. La propuesta de von Polenz es más amplia que la de Helbig (1979: 70), en la que no se contempla el valor [+pasivo].

32 Cf. Helbig (1979: 275) y von Polenz (1987: 175).

33 Cf. Daniels (1963: 16), Heringer (1968: 26-28), Helbig (1979: 274), von Polenz (1987: 171), etc. 
eventivo tal y como se expone en relación al atributo primario [B], ya que, esta distinción no siempre se fundamenta en propiedades lingüísticas ${ }^{34}$.

Por otra parte, también en consonancia con la expresión del evento, muchos estudios aluden a que el sustantivo deriva de un verbo o adjetivo, aunque, por lo general, no suele considerarse un criterio restrictivo ${ }^{35}$. Este planteamiento presenta ciertas dificultades interpretativas, principalmente en relación al tratamiento genérico del fenómeno de la derivación y la dificultad para determinar la direccionalidad de los procesos derivativos. Pensemos, por ejemplo, en una construcción como Geschäfte machen. El sustantivo, según la definición del DUW, expresa un evento: "auf Gewinn abzielende [kaufmännische] Unternehmung, [kaufmännische] Transaktion; Handel”. Este sustantivo, en Mittelhochdeutsch gescheft con el significado de "Beschäftigung, Arbeit, Angelegenheit; Anordnung, Befehl; Testament; Abmachung, Vertrag" (DHW), es un derivado del verbo schaffen: "zustande bringen, tätig sein [...] arbeiten" (DHW). El sustantivo Geschäft es, por lo tanto, un sustantivo deverbal en sentido estricto, aunque la relación semántica entre este en una construcción como Geschäfte machen y el verbo base de la derivación es poco transparente desde un punto de vista sincrónico.

En nuestra propuesta, de acuerdo con Heringer (1968: 27), von Polenz (1987: 175) y Kamber (2008: 26), relativizamos la importancia de la dimensión diacrónica en la configuración del ejemplar prototípico de FVG. El factor determinante es que el sustantivo presente una estructura morfológica transparente que nos permita establecer vínculos semánticos con su base léxica, independientemente de que esta se corresponda o no con su base etimológica. Este planteamiento se fundamenta en la noción de derivación enmarcada en el dominio de la morfología sincrónica y, así, en "la conexión de significados que se da en la conciencia lingüística de los hablantes" (NGLE, 2009: 342). Bajo esta premisa, una construcción como Geschäfte machen se interpreta como un FVG adscrito al entorno periférico del paradigma.

En cuanto a la direccionalidad del proceso derivativo y en consonancia con la tendencia general en el estudio de los FVG en lengua alemana ${ }^{36}$, el factor que determina el carácter prototípico de la construcción es la coexistencia en el sistema lingüístico del sustantivo y un verbo o adjetivo, que, además de compartir base léxica, mantengan una relación semántica transparente. Bajo esta premisa, construcciones como in den Selbstmord treiben formarían parte del entorno periférico, mientras que otras como ins Visier nehmen, de cuyo sustantivo deriva el verbo visieren, se interpretan como ejemplares prototípicos $^{37}$. Esta forma de proceder responde a un fin pragmático en el que prima lo semántico sobre lo etimológico y lo sincrónico sobre lo diacrónico.

[E] El componente nominal es un grupo preposicional: el carácter prototípico de las construcciones con componente nominal preposicional frente a aquellas con un componente nominal en acusativo se fundamenta tradicionalmente en base a su mayor grado de fijación, materializado, tal y como afirma Helbig (1979: 275), en la neutrali-

34 Cf. NGLE (2009: 796). La problemático de la distinción entre sustantivos abstractos y concretos se recoge también en la gramática Duden (2016: 174).

35 Cf. Daniels (1963: 15), Helbig (1979: 276), Heringer (1968: 26- 27), von Polenz (1987: 169- 170) y Kamber (2008: 26).

36 Véanse, entre otros, Heringer (1968: 27), von Polenz (1987: 175), Tao (1997: 41ss.), Seifert (2004: 211) y Kamber (2008: 26).

37 Que un sustantivo comparta estructura morfológica con un verbo o adjetivo no implica la existencia de una derivación transformacional entre el FVG y ese verbo o adjetivo (in Frage stellen $\neq$ fragen). De igual forma, la relación semántica de un FVG con un verbo pleno parafrástico no implica la existencia de una relación morfológica entre ese verbo y el sustantivo de la construcción verbonominal (in Frage stellen = zweifeln). 
zación de una serie de mecanismos morfosintácticos como los descritos en el apartado 2 ( $c f$. Tabla 1), de mayor incidencia en los FVG con componente nominal en acusativo. Otro argumento que refuerza el carácter prototípico del componente nominal preposicional se pone de manifiesto en relación con el ejemplo (9), en el que, aparentemente, la variante plena y funcional de un verbo se activan simultáneamente en una misma unidad funcional. En este supuesto, los FVG con componente nominal en acusativo parecen favorecer la omisión de una de las variantes en el contexto oracional, lo que puede suceder en consonancia con una percepción de este tipo de FVG próxima a las combinaciones libres con verbo pleno transitivo. Por el contrario, en el caso de los FVG con componente nominal preposicional, ejemplos del tipo ich komme in Bewegung und ins Kino, en el que se omite una de las variantes del verbo, funcional o plena, no parecen plausibles, lo cual, principalmente, se debe al carácter desemantizado, que, junto al verbo, también presenta la preposición ${ }^{38}$ y que, desde nuestro punto de vista, puede favorecer la percepción diferenciada de estas construcciones respecto a las construcciones con verbo pleno y complemento circunstancial de dirección.

La combinación de los atributos primarios y secundarios tiene como resultado la configuración del siguiente paradigma:

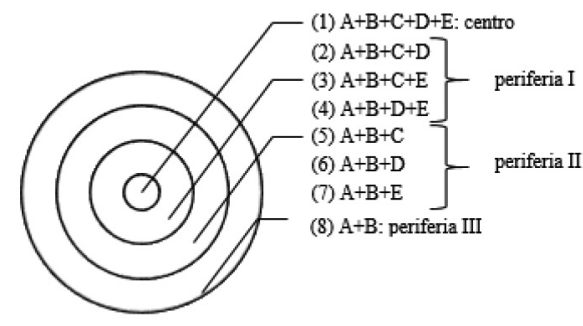

Imagen 3. Representación del paradigma de FVG a partir de la combinación de los atributos primarios y secundarios.

(1) $\mathrm{A}+\mathrm{B}+\mathrm{C}+\mathrm{D}+\mathrm{E}$ : zur Anwendung kommen

(2) $\mathrm{A}+\mathrm{B}+\mathrm{C}+\mathrm{D}:$ Abschied nehmen

(3) $\mathrm{A}+\mathrm{B}+\mathrm{C}+\mathrm{E}$ : zum Zug kommen

(4) $\mathrm{A}+\mathrm{B}+\mathrm{D}+\mathrm{E}$ : zum Kauf bewegen

(5) $\mathrm{A}+\mathrm{B}+\mathrm{C}$ : Geschäfte machen

(6) $\mathrm{A}+\mathrm{B}+\mathrm{D}$ : Information weiterleiten

(7) $\mathrm{A}+\mathrm{B}+\mathrm{E}$ : in den Selbstmord treiben

(8) A+B: eine Maßnahme ergreifen

El grupo (1) se sitúa en el centro del paradigma y constituye el ejemplar prototípico, el mejor representante de la categoría en el que se combinan la totalidad de atributos primarios y secundarios. El resto de grupos se ubican en el entorno perifé-

38 Como en el caso de los VF, la desemantización de la preposición también se valora en términos graduales. En relación a la semántica de la preposición del componente nominal y su aportación al conjunto de la construcción véanse, entre otros, Heringer (1968: 41), Persson (1975: 67- 68) y Seob So (1991: 31- 32). 
rico, en el que se diferencian tres niveles según el número de atributos secundarios operativos. En el primer nivel, denominado periferia I, se sistematizan las construcciones adscritas a los grupos (2), (3) y (4). Se trata de FVG en los que se activan dos de los tres atributos secundarios formulados $\mathrm{y}$, por lo tanto, de las construcciones del entorno periférico más próximas al ejemplar prototípico. En el segundo nivel, periferia II, se sistematizan también tres grupos de construcciones, (5), (6) y (7), que tan solo activan uno de los atributos secundarios formulados. Por último, en el tercer nivel, periferia III, se sitúan los FVG más distantes del ejemplar prototípico, ya que solo activan los atributos primarios.

\section{Conclusiones}

Nuestra propuesta de representación paradigmática de los FVG en torno a la configuración de un ejemplar prototípico y su entorno categorial ofrece una delimitación de las construcciones a partir de la intersección de varios parámetros que actúan de forma simultánea. Esta forma de proceder pone de manifiesto el carácter heterogéneo del paradigma en consonancia con la naturaleza no discreta de la categoría lingüística más allá del grado de operatividad de una serie de restricciones morfosintácticas.

La idea subyacente es que este tipo de restricciones, formuladas a partir de la realización de test de conmutación, permutación y paráfrasis en el marco de la metodología estructural-funcionalista, permiten caracterizar el comportamiento de los FVG en un contexto determinado e incluso valorar su proximidad a los paradigmas vecinos en base a su grado de fijación; sin embargo, no determinan cuál es el mejor representante de su clase a partir de la descripción de las construcciones dentro del propio paradigma e independientemente del contexto en el que se realicen. El modelo de representación paradigmática aquí propuesto es, por lo tanto, una abstracción que permite delimitar el objeto de estudio a partir de una serie de parámetros que responden a la propia naturaleza de las construcciones como unidades de significado en el sistema general de la lengua alemana, sin rechazar por ello la existencia de posibles variaciones en su realización.

La diferencia fundamental con el modelo de Kamber (2008) reside en la formulación de un requisito básico de formación relativo al componente nominal, el atributo primario [B], que, desde nuestro punto de vista, responde de forma más adecuada a la percepción diferenciada de las construcciones como una única unidad de significado al poner de relieve la relación de interdependencia mutua entre el verbo y el sustantivo en la expresión del predicado. Por otra parte, y atendiendo a la formulación de los atributos, se observa que los atributos primarios, como sucede en los modelos taxonómicos clásicos, responden a una valoración binaria, lo que, a priori, podría interpretarse como una contradicción respecto a la concepción no discreta del paradigma. La diferencia reside en que, como apuntamos anteriormente, estos atributos se valoran de forma simultánea y no de forma individual.

Para concluir consideramos que, pese a la dificultad que entraña la parametrización de categorías no discretas, nuestra propuesta de representación paradigmática es adecuada para la delimitación del objeto de estudio. En un nivel más profundo de especificación, la conceptualización del ejemplar prototípico y ejemplares periféricos resultantes permite llevar a cabo un análisis sobre el perfil morfosintáctico de las 
construcciones a partir de la observación de su comportamiento en contextos comunicativos concretos, que, por ejemplo, pueden ponen de manifiesto particularidades o variaciones en su realización morfosintáctica, pero que, en nuestro planteamiento, constituyen el fin de la investigación y no el punto de partida como parámetros de delimitación del fenómeno lingüístico.

\section{Referencias bibliográficas}

Alonso Ramos, M., Las construcciones con verbos de apoyo. Madrid: Visor 2004.

Bahr, B. I., Untersuchungen zu Typen von Funktionsverbfügungen und ihrer Abgrenzung gegen andere Arten der Nominalverbindung. Bonn: Universität Bonn 1977.

Blum, S., «Ahd. habên in Funktionsverbgefügen», Beiträge zur Erforschung der deutschen Sprache (1986), 80- 95.

Bresson, D., «La distribution du sens dans les locutions à verbe support», Cercle linguistique d'Aix-en-Provence 7. La signification (1989), 57- 72.

Castell, A., «Funktionsverbgefüge im Deutschen, Spanischen und Katalanischen», Forum 9 (1999), 125-133.

Daniels, K., Substantivierungstendenzen in der deutschen Gegenwartssprache. Düsseldorf: Schwann 1963.

De Miguel, E., «El aspecto léxico», en: Bosque, I. y Demonte, V (eds.), Gramática descriptiva de la lengua española. Madrid: Espasa 1999, 2979- 3060.

Detges, U., Untersuchungen zur Syntax und Semantik französischer Funktionsverbfügugen (Masch. Staatsexamensarbeit). Freie Universität Berlín 1985.

Detges, U., Nominalprädikate: eine valenztheoretische Untersuchung der französischen Funktionsverbgefüge des Paradigmas - être Präposition Nomen - und verwandter Konstruktionen. Tübingen: Niemeyer 1996.

DHW: Das Herkunftswörterbuch. 3., völlig neu bearbeitete und erweiterte Auflage. Mannheim/ Leipzig/ Viena/ Zurich: Dudenverlag 2001.

Duden, Der große Duden. Grammatik der deutschen Gegenwartsprache. 1., Auflage. Mannheim: Dudenverlag 1959.

Duden, Der große Duden. Grammatik der deutschen Gegenwartsprache. 2., vermehrte und verbesserte Auflage (Hrsg. P. Grebe). Mannheim: Bibliographisches Institut 1966.

Duden, Der große Duden. Grammatik der deutschen Gegenwartsprache. 3., bearbeitete und erweitete Auflage (Hrsg. P. Grebe). Mannheim: Bibliographisches Institut 1973.

Duden, Die Grammatik. 9., vollständig überarbeitete und aktualisierte Auflage. Mannheim/ Zurich: Dudenverlag 2016.

DUW: Deutsches Universalwörterbuch. 6., überarbeitete und erweitete Auflage. Mannheim/ Leipzig/ Viena/ Zurich: Dudenverlag 2006.

Egido Vicente, M., El tratamiento teórico-conceptual de las construcciones con verbos funcionales en la tradición lingüistica alemana y española. Frankfurt am Main: Peter Lang 2016.

Eisenberg, P., Grundriss der deutschen Grammatik. Der Satz. 4., aktualisierte und überarbeitete Auflage.Stuttgart/ Weimar: Metzler 2013.

Engel, E., Sprich Deutsch! Zum Hilfsdienst am Vaterland. Leipzig: Hesse \& Becker 1917.

Engel, U., Deutsche Grammatik. Neubearbeitung. Múnich: Iudicum 2004.

Engelen, B., «Zum System der Funktionsverbgefüge», Wirkendes Wort 18 (1968), 289-303.

Fix, U., (1974-1976) «Zum Verhältnis von Syntax und Semantik im Wortgruppenlexem» Beiträge zur Geschichte der deutschen Sprache und Literatur 95 (1974), 214- 318; (1976) 7-78. 
Gerling, M. / Orthen, N., Deutsche Zustands- und Bewegungsverben. Eine Untersuchung zu ihrer semantischen Struktur und Valenz. Tübingen: Narr 1979.

Götze, L., «Funktionsverbgefüge im Deutschunterricht für Ausländer», Zielsprache Deutsch 2 (1973), 54- 61.

Götze, L., Valenzstrukturen deutscher Verben und Adjektive: Eine didaktische Darstellung für das Fach Deutsch als Fremdsprache. Múnich: M. Hueber Verlag 1979.

Günther, H. / Pape, S., «Funktionsverbgefüge als Problem der Beschreibung komplexer Verben in der Valenztheorie», en: Schumacher, H. (ed.), Untersuchungen zur Verbvalenz. Tübingen: Narr 1976, 92- 128.

Heine, A., Funktionsverbgefüge in System, Text und korpusbasierter (Lerner-) Lexikographie. Frankfurt am Main: Peter Lang 2006.

Helbig, G., «Probleme der Beschreibung von Funktionsverbgefügen im Deutschen», Deutsch als Fremdsprache 16 (1979), 273-285.

Hentschel, E ./ Weydt, H., Handbuch der deutschen Grammatik. 4., vollständig überarbeitete Auflage. Berlín/ Boston: de Gruyter 2013.

Heringer, H. J., Die Opposition von „kommen“ und „,bringen“ als Funktionsverbgefüge. Untersuchungen zur grammatischen Wertigkeit und Aktionsart. Düsseldorf: Schwann 1968.

Herrlitz, W., Funktionsverbgefüge von Typ ,, in Erfahrung bringen “. Ein Betrag zur generativ-transformationellen Grammatik des Deutschen. Tübingen: Niemeyer 1973.

Kamber, A. / Näf, A., «Locutions verbales en français- Funktionsverbgefüge en allemand: essai de bilan descriptif et terminologique», en: Colomba, B. y Savelli, M. (eds.), Métalangage et terminologie linguistique. Actes du colloque international de Grenoble. Leuven: Peeters 2001, 901- 913.

Kamber, A., Funktionsverbgefüge-empirisch. Eine korpusbasierte Untersuchung zu den nominalen Prädikaten des Deutschen. Tübingen: Niemeyer 2008.

Kleiber, G., La sémantique du prototype. París: PUB 1990.

Koch, P., Verb - Valenz - Verfügung. Zur Satzsemantik und Valenz französischer Verben am Beispiel der Verfügungsverben. Heidelberg: C. Winter 1981.

Kolb, H., «Sprache des Veranlassens», Sprache in technischen Zeitalter 5 (1962), 372-387.

Korn, K., «Sprache in der verwalteten Welt», Sprache in technischen Zeitalter 5 (1962), 365-372.

Lakoff, G., Women, Fire, and Dangerous Things. Chicago: University of Chicago Press 1987.

Lipka, L., «Lexicalization and institutionalization in English and German», Linguistica Pragensia 2 (1992), 1-13.

Mendívil Giró, J. L., Las palabras disgregadas. Sintaxis de las expresiones idiomáticas y los predicados complejos. Zaragoza: Prensas Universitarias de Zaragoza 1999.

Moure, T., «La teoría de los prototipos y su aplicación en gramática (I y II)», Contextos XII (1994), 167- 219.

NGLE: Nueva gramática de la lengua española. Madrid: Espasa 2009.

Persson, I., Das System der kausativen Funktionsverbgefüge. Eine semantisch-syntaktische Analyse einiger verwandter Konstruktionen. Lund: CWK Gleerup 1975.

Porzig, W., (1930): «Die Leistung der Abstrakta in der Sprache», Blätter für deutsche Philosophie 4 (1930), 66-77.

Relleke, W., «Funktionsverbgefüge in der althochdeutschen Literatur», Amsterdamer Beiträge zur Älteren Germanistik 7 (1974), 1- 46.

Reuther, T., «On Dictionary Entries for Support Verbs: The Cases of Russian VESTI, PROVODIT and PROIZVODIT», en: Wanner, L. (ed.), Recent Trends in Meaning-Text Theory. Ámsterdam/ Philadelphia: Benjamins 1996, 181- 208. 
Rosch, E., «Natural Categories», Cognitive Psychology 4 (1973), 328- 350.

Rösch, O., Untersuchungen zu passivwertigen Funktionsverbgefügen im Deutschen der Gegenwart. Hamburgo: Buske 1994.

Rothkegel, A., Feste Syntagmen. Grundlagen, Strukturbeschreibung und automatische Analyse. Tübingen: Niemeyer 1973.

Schemann, H., «Zur Integration der Funktionsverbgefüge in die Idiomatikforschung», Deutsche Sprache 10 (1982), 83- 96.

Seifert, J., Funktionsverbgefüge in der deutschen Gesetzessprache (18.-20. Jahrhundert). Hildesheim/ Zurich/ Nueva York: Olms 2004.

Seob-So, M., Die deutschen Funktionsverbgefüge in ihrer Entwicklung vom 17. Jahrhundert bis zur Gegenwart. Trier: WVT 1991.

Starke, G., «Zum Einfluß von Funktionsverbgefügen auf den Satzbau im Deutschen», Deutsch als Fremdsprache 3 (1975), 157- 163.

Sütterling, L., Die deutsche Sprache der Gegenwart. Ein Handbuch für Lehrer und Studierende. Leipzig: Voigtländer 1907.

Tao, J., Mittelhochdeutsche Funktionsverbgefüge. Tübingen: Niemeyer 1997.

von Polenz, P., «Funktionsverben im heutigen Deutsch. Sprache in der rationalisierten Welt», Wirkendes Wort 5 (1963), 5- 46.

von Polenz, P., «Funktionsverben, Funktionsverbgefüge und Verwandtes. Vorschläge zur satzsemantischen Lexikografie» Zeitschrift für germanistische Linguistik 15 (1987), 169- 189.

Weiskopf, F. C., Verteidigung der deutschen Sprache. Berlín: Aufbau Verlag 1955.

Wierzbicka, A., «Prototypes save: on the cases and abuses of the notion of prototype in Linguistics and related fields», en: Tsohatzidis, S. L. (ed.), Meanings and Prototypes, Oxford: Routledge 1989, 347- 367.

Zifonun, G. et al., Grammatik der deutschen Sprache. Berlín/ Nueva York: de Gruyter 1997. 